\title{
1 Assessing Genome-Wide Significance for the Detection of Differentially
}

\section{Methylated Regions}

3 Christian M. Page ${ }^{1,2,3, *, 9}$, Linda Vos ${ }^{4, *}$, Trine B. Rounge ${ }^{4,5}$, Hanne F. Harbo ${ }^{1,2}$, Bettina K. Andreassen ${ }^{4, \S}$ 4

${ }^{1}$ Department of Neurology, Institute of Clinical Medicine, Faculty of Medicine, University of Oslo, Norway

${ }^{2}$ Department of Neurology, Division of Clinical Neuroscience, Oslo university hospital, Norway

$9{ }^{3}$ Department of non-communicable diseases, Norwegian Institute of Public Health, Norway

$10{ }^{4}$ Department of Research, Cancer Registry of Norway, Oslo, Norway

$11{ }^{5}$ Genetic epidemiology group, Folkhälsan research center, Helsinki, Finland

12 s Corresponding author; email: B.K.Andreassen@kreftregisteret.no

13 *Equal contributions

14 "Current adress: Oslo Centre for Biostatistics and Epidemiology, Oslo University Hospital, Oslo, 15 Norway 


\section{Abstract}

Motivation: DNA methylation plays an important role in human health and disease, and methods for the identification of differently methylated regions are of increasing interest. There is currently a lack of statistical methods which properly address multiple testing, i.e. control genome-wide significance for differentially methylated regions.

Methods: We introduce a scan statistic (DMRScan), which overcomes these limitations. We benchmark DMRScan against two well established methods (bumphunter, DMRcate), using a simulation study based on real methylation data. An implementation of DMRScan is available from Bioconductor.

Results: Our method has higher power than alternative methods across different simulation scenarios, particularly for small effect sizes. DMRScan exhibits greater flexibility in statistical modeling and can be used with more complex designs than current methods.

Conclusion: DMRScan is the first dynamic approach which properly addresses the multipletesting challenges for the identification of differently methylated regions. DMRScan outperformed alternative methods in terms of power, while keeping the false discovery rate controlled.

\section{Keywords}

34 Differentially methylated regions; Scan statistics; Sliding window; Genomics

\section{Introduction}

DNA methylation is an epigenetic marker, which can explain variation in gene expression, as

37 well as cell differentiation and other variability in cell phenotypes[1-3]. It is the most studied 
epigenetic modifier on a genome-wide scale[4]. DNA methylation is believed to play an important role in the pathology of complex diseases. In cancer, large changes in the global methylation level have been observed[5]. However, for most other complex diseases, there has been little evidence of such a global change in DNA methylation. This has led to the notion that local methylation differences in smaller regions (called differentially methylated regions; DMRs) may be relevant for these diseases[6]. Although methylation at specific CpG sites may have an effect on its own[7], it is often assumed that multiple methylation sites within a cluster of CpGs are involved in a change of cell characteristics[3]. Several CpGs within a region might contribute to a disease or phenotype, but their individual effects may not be strong enough to pass a genomic-wide significance threshold. In recent genome-wide methylation studies, there has been an increasing focus on identifying significant DMRs by combining methylation information from neighboring $\mathrm{CpG}$ sites[8]. The underlying thought is to increase power by reducing the requirements for multiple testing adjustments through accumulation of correlated signals.

There are two types of procedures for determining DMRs. The first procedure is based on underlying biological knowledge with respect to the unit of interest. For instance, the CpG sites can be grouped by their affiliation to genes, regulatory regions, $\mathrm{CpG}$ islands or pathways. These fixed units can be analyzed separately with respect to the phenotypes of interest, and the units are classified as DMRs if there is enough evidence for association. Multiple testing procedures can be easily applied by taking into account the number of predetermined regions. The second type of aggregation is dynamic, where the borders are not pre-determined, but rather data driven, as $\mathrm{CpG}$ sites in close proximity are collapsed into regions in order to identify potential DMRs. Adjustment for multiple testing when using this approach is challenging and still developing. 
Several methods have been proposed to identify DMRs, such as BSmooth, bumphunter, Comb-p, DMRcate, dmrseq, DMRMark, and ProbeLasso [2, 9-13]. Additionally, there are methods (csaw and PeakSeq $[14,15])$ for peak detection involving ChIP-seq data, thus relying on count data. The underlying theory, however, could also be applied to DNA methylation data. Many of these methods are tailored to a specific technology (e.g. dmrseq, DMRMark, BSmooth and ProbeLasso), while some are compatible with almost any measurement technology (bumphunter, Comb-p, and DMRcate). Applying peak detection methods for ChIP-seq on methylation data requires non-trivial adaptations and is outside the scope of this paper. We selected methods based on dynamic aggregation, identifying DMRs which are independent of technology and appropriate to use for both sequencing and chip data. This excludes static methods such as ProbeLasso and methods only applicable to one specific technology, such as dmrseq, BSmooth, and DMRMark. Two widely used methods meeting these criteria were selected for comparison purposes to our method; bumphunter and DMRcate[2, 10]. The bumphunter algorithm is among the most commonly used approach when interrogating DMRs and can be considered as the "gold standard" for DMR calling. The peak calling packages are mostly directed towards ChIP-seq data, and the input data are often structured differently than for methylation data; as such it is difficult to apply directly to methylation data without modifying the source code.

Bumphunter was among the first methods that proposed a multiple-testing adjusted procedure when scanning the epigenome for significant regions[10]. Bumphunter's multiple testing adjustment for the region p-values considers regions where the effect sizes exceed a threshold. There are two ways to adjust the p-values for the selection step, either by permuting the casecontrol status or with Monte Carlo simulation from a truncated multivariate normal distribution of the same size as the detected region [16]. DMRcate reports a minimum p-value 
within a region as well as an aggregated p-value based on Stouffers method[17]. Both these pvalues can be hard to interpret, and do not necessarily keep the overall $\alpha$-level.

There is a wide range of literature on scan statistics, which is based on extreme value theory and uses a well-defined theoretical framework, which allows us to overcome the limitations of current methods and to identify genome-wide significant DMRs. Our introduced method, DMRScan, properly adjusts for multiple testing by keeping the false positives controlled at the $\alpha$-level significance threshold. Several variants of scan statistics have been successfully applied on different types of genomic data[5, 7]. We propose an adoption of a sliding window approach previously used in peak detection for ChIP-chip tiling arrays[18]. Despite of some similarity to the csaw R-package [14], there are notable differences. The csaw method addresses the issue of FDR control by combining locus-wise $p$-values to a region-wise $p$-value using Simes' method. The region-wise p-values are adjusted using a Benjamini-Hochberg FDR correction, while our method relies on Poisson heuristics to assess genome wide significance.

\section{Material and Methods}

\section{Bumphunter and DMRcate}

Bumphunter[10] identifies all CpG sites over a certain percentile of the test statistic distribution (cut-off parameter). These sites are aggregated together into clusters based on their genomic position. Region-wise p-values are estimated using either permutation or bootstrap approaches. By permuting the outcome variable, a set of null regions are constructed. The candidate regions are compared with the distribution of the null regions in both length and area under the curve. The proportion of null regions with an area under the 
108 curve and a region length being at least as extreme as the candidate region is presented as the

109 family-wise error rate for the given region.

110 DMRcate[2] applies a Gaussian kernel smoothing on the site-wise test statistic, after using a

111 limma model[19] on each CpG. Using the method of Satterthwaite[20], probe-wise p-values

112 are calculated for the smoothed test statistic. After adjustment for multiple testing (by FDR),

113 nearby genome-wide significant probes are aggregated into regions. Using Stouffer's

114 method[17] on the adjusted probe-wise p-values, a region-wise p-value is calculated using all

115 probes within the candidate regions.

\section{$116 \underline{\text { DMRScan }}$}

117 DMRScan is a sliding window approach based on extreme value theory, which has earlier

118 been applied to peak detection for transcription factor binding sites[18]. It is based on the

119 observation from Aldous[21], that for a large enough threshold, the number of significant

120 windows in a scan statistic surpassing the threshold will follow a Poisson distribution.

121 Using extreme value theory, Zhang deduced a relationship between the significance level $(\alpha)$

122 and the intensity of the Poisson distribution $(\lambda)$ for the number of peaks above a threshold.

123 Assuming independent tests, we get that: $\alpha=1-\mathrm{e}^{-\lambda}$. By putting a constraint that no two

124 overlapping windows can both be significant, Zhang constructs independent observations. A

125 natural extension of this is to use different window sizes. To create independent observations,

126 nested or overlapping windows cannot both be significant. In such a case, the smallest

127 window would be regarded as the significant window[18].

128 The intensity $(\lambda)$ is dependent on the window threshold $(t)$, the correlation structure of the test

129 statistics, and the window size (k). Using a Monte Carlo simulation with different thresholds, 
130 Zhang was able to derive a relationship between the threshold and the significance level of the

131 test for each window size[18].

132 For every $\mathrm{CpG}$ site, a linear regression analysis was done with methylation level as the

133 dependent variable and case-control status as the explanatory variable. However, there are no

134 restrictions with respect to the statistical model used on each $\mathrm{CpG}$ site in order to determine

135 the probe wise statistic. Different link functions can be chosen and additional explanatory or

136 confounding variables can be added with little computational cost. Hence, one is able to select

137 a statistical model which fits the data best. The $\mathrm{CpG}$ wise test statistic will be denoted as $\mathrm{T}_{\mathrm{CpG}}$.

138 For each window-size $k$, we used Monte Carlo simulation to determine the minimal threshold

$139 t_{k}$ based on the significance level $\alpha$. We chose the window threshold $\left(t_{k}\right)$ of the window

140 statistic $\left(\mathrm{T}_{\mathrm{DMR}}\right)$ such that the expected number of significant tests $\left(\mathrm{E}_{\mathrm{k}}\right)$ for each window size $k$

141 was equal (see Appendix 1, eq. 2).

142 Three variants of DMRScan using different methods to determine the window thresholds $t_{k}$

143 were implemented: DMRScan (MCMC), DMRScan (Importance sampling) and DMRScan

144 (Siegmund). In the first two approaches, a Monte Carlo simulation is used to determine the

145 threshold given the dependency structures for the $\mathrm{T}_{\mathrm{CpG}}$ 's. For DMRScan (Siegmund), the

146 thresholds are calculated using an analytic expression.

147 In DMRScan $(M C M C)$, a Monte Carlo simulation was used to determine the number of

148 significant tests over the threshold. In this algorithm, one is free to choose the optimal model

149 for the dependency structure of the test statistic $\mathrm{T}_{\mathrm{CpG}}$ based on the underlying data.

150 DMRScan (Importance sampling) uses a local average of independent Gaussian variables to

151 describe the dependency structure of the statistic $\mathrm{T}_{\mathrm{CpG}}$, assuming a dependency of two probes

152 in both directions. Properties of the standard normal distribution in a fast importance sampling 
153 algorithm were used to simulate the intensity of the number of windows exceeding the

154 threshold. Importance sampling was over 700 times faster than the MCMC algorithm.

155 A modification of Zhang's method was introduced by Siegmund et al.[22] and implemented 156 in DMRScan (as the option "Siegmund" in the DMRScan function call). Here, the intensity

157 for the Poisson distribution $(\lambda)$ is analytically calculated as a function of the desired threshold.

158 This derivation is based on the assumption that the test statistic follows an Ornstein-

159 Uhlenbeck process (OU-process). A closed form solution was first published by

160 Siegmund[23] and later re-formulated in[24] [pp. 112],

161

$$
\lambda=2 \beta L t_{k} \phi\left(t_{k}\right) v\left(t_{k}(2 \beta \Delta)^{1 / 2}\right)
$$

162 Here $\lambda$ is the intensity of windows over the threshold $\left(t_{k}\right), L$ is the genetic length of the

163 chromosome (in number of CpGs), $\beta=1 / k$ is the autoregressive parameter of the OU-process

164 where $k$ is the window size, $\Delta$ is the spacing between observations (assumed to be 1 ). The

165 function $v($.$) can be approximated by$

$$
v(y) \approx \frac{(2 / y)(\Phi(y / 2)-0.5)}{(y / 2) \Phi(y / 2)+\phi(y / 2)}
$$

167 The functions $\Phi($.$) and \phi($.$) are the cumulative distribution and the density function of the$ 168 standard normal distribution, respectively.

169 Multiple-testing adjusted p-values for the genome-wide significant DMRs can be derived by a 170 combination of empirics and theoretical properties. The variance of the test statistic of the

171 window of interest with window size $k$ is approximated using simulation and theoretical

172 asymptotic p-values are derived using the standard normal distribution (see Appendix, eq 3).

173 Alternatively, empirical p-values can be calculated by comparing the value of the test statistic 
$174 T_{\text {DMR }}$ for the window of interest of window size $k$ with the distribution of all test statistics

$175 \mathrm{~T}_{\mathrm{DMR}}$ for windows with the same window size $k$.

176 DMRScan, together with an example dataset is implemented as an R library in Bioconductor.

177 An illustrating example of its usage is given in the supplementary material to this paper.

\section{Results}

179 Simulation study

\section{$180 \quad$ Procedure}

181 We used real methylation data from chromosome 22 from the Finnish Health in Teens study

182 (Fin-HIT, http://www.finhit.fi/for-researchers/), described in more detail here [25]. The

183 backbone for the $\mathrm{CpG}$ regions was known $\mathrm{CpG}$ regions at chromosome 22. To evaluate and

184 compare the methods, we tested them on 100 causal regions. This number is a trade-off

185 between few regions (biological plausibility) and having an extensive testing of the methods

186 (many regions). We let the frequency of the causal region be inversely proportional to its

187 length, thus shorter regions were more frequent than longer regions in the simulation. We

188 added an effect by changing the methylation beta-values[26] of the causal CpGs such that the

189 mean difference between cases and controls in that region were equal to the effect size. The

190 beta-values are ranging from 0 to 0.15 and always within the legal limit of 0 to 1 . The first

191 simulation was on the original data set with no added effect. The causal regions ranged in size

192 from 5 to 100 sequential $\mathrm{CpG}$ sites, reflecting the range which seems biologically relevant and

193 plausible [27]. A CpG island could not have more than one causal region and the maximum

194 distance between the causal CpGs could not exceed the maxGap parameter in all methods. 
195 In each causal region, we added an artificial effect and compared the performance in retrieval

196 of these 100 regions for the five methods (i) bumphunter, (ii) DMRcate, (iii) DMRScan

197 (MCMC) with thresholds based on extreme value theory using Monte Carlo simulation, (iv)

198 DMRScan (Importance Sampling), where an importance sampling algorithm was used to

199 determine the thresholds, (v) and DMRScan (Siegmund), with an analytic expression was

200 used to determine the window thresholds.

201 For each effect size, we counted the number of true positive and false positive DMRs (Figure

2021 A-B). Any DMRs overlapping with a causal region was counted as true positive

203 observation. We also summed the number of significant probes in each DMR, occurring both

204 inside and outside of the causal regions (Figure 1 C-D). Hence, the number of true and false

205 discoveries from both a DMR and CpG perspective were gathered.

\section{DMRScan}

207 When inspecting the test statistics $\mathrm{T}_{\mathrm{CpG}}$ on a subset of the data, an $\mathrm{AR}(2)$ process gave the

208 best description of the dependence structure in our subset. Hence we used an AR(2) process

209 as a null model to determine the thresholds in DMRScan (MCMC).

210 For window thresholds between 0.8 to 4 with regular increments of 0.2 , and different window

211 sizes $(k)$ from 2 to 10 , we simulated test statistics from a null model and applied DMRScan

212 with fixed window size and no overlapping significant windows. We determined the number

213 of significant windows for the different window sizes and thresholds. This was done using

214 both the MCMC and the Importance sampling approach. For the different window sizes $(k)$,

215 we chose the window threshold $\left(t_{k}\right)$ such that the expected number of significant tests

216 E[significant.window] was equal for all window sizes (see Appendix, eq. 2). Since we placed 
217 the different thresholds on a grid, interpolation was used to determine the minimal threshold

218 keeping the significance level $\alpha$ at a genome wide significance level.

219 Using the analytic formula of Siegmund, we calculated thresholds $t_{k}$ for each window size $k$

220 such that the expected number of significant tests $\mathrm{E}$ [significant.window] is equal for all

221 window sizes (see Appendix, eq. 2). A detailed explanation for the parameter choices is given

222 in the supplementary materials and methods, and a full list of our parameter choices is listed

223 in Table 1.

\section{Power assessment}

225 We define the power as the proportion of true, genome wide significant causal DMRs. The

226 number of true positive and false positive regions is shown in Figure 1 (A and B), as a

227 function of increasing effect size. All three versions of the DMRScan algorithm had a faster

228 convergence in power compared to bumphunter when calling DMRs. DMRcate outperformed

229 Bumphunter in DMR calling, however, this came at a cost of a higher number of false

230 positive probes (Figure $1 \mathrm{C}$-D). The false positive probes in DMRcate were in close proximity

231 of the causal regions, but the proportion of false positive probes was considerable as

232 compared to the other methods.

233 Since the thresholds for the sliding windows are static, the false discovery rate for DMRScan

234 was independent of the added effect size and remained fixed throughout the simulations

235 (Figure 1 B). The number of false positive of DMRscan(siegmund) was approximately equal

236 to that of Bumphunter. For DMRcate, the number of false positive sites increased with

237 increasing effect size, this can be seen in Figure 1(D). On closer investigation, all of the

238 reported false discoveries lay on the edges of a causal region, and no false positive regions

239 independent of any causal DMRs were detected. The false positive discoveries were due to 
DMRcate's smoothing effect on the border of the regions, where the smoothing extended the

241 reported regions beyond the causal part. DMRScan with a theoretically derived threshold 242 using Siegmund's model had the lowest false positive rate, which was close to zero. The 243 importance sampling threshold had a marginally higher false positive rate, but a substantially 244 faster convergence in true positives.

245 We observe the biggest difference between the methods for small effect sizes. Bumphunter 246 had a negligible proportion of true positives for effect sizes under 0.05 , while the sliding 247 windows and DMRcate were much more responsive for small effect sizes. DMRcate tended 248 to have a higher false positive rate than the sliding windows approaches, even for very low 249 effect sizes. For the DMRScan with importance sampling and Monte Carlo thresholds, the 250 number of false positive observations was small. Three and 5 of 971 regions $(0.5 \%)$ were 251 falsely detected, respectively.

\section{Discussion}

253 We have proposed a new method for identifying DMRs, based on Poisson heuristics and a 254 sliding window approach. We compared this to other established methods for identifying 255 DMRs. The approach introduced in this paper is based on an approach presented by Zhang 256 which was originally introduced for ChIPseq analysis. With some modifications, it is now 257 applicable to DNA methylation analysis. However, the method itself may not be restricted to 258 those two areas. Scan statistics can be used for peak detection on any data containing 259 correlated observations.

260 For most complex diseases, $\mathrm{CpG}$-wise test statistics are not likely to contain distinct peaks 261 like those observed in ChIP-seq. Thus, the thresholds for the region wise test statistics have to 262 be very close to the observed test statistic, $\mathrm{T}_{\mathrm{DMR}}$, in order to pick up any signals. When the 
263 threshold lies close to the observed test statistic, the number of false positive windows will be

264 very sensitive to small changes in the threshold.

265 Having 100 causal regions in one analysis is quite optimistic, but was chosen to provide a 266 good spread on the different length of causal DMRs while maintaining computational 267 efficiency. Longer DMRs were assumed rare and few causal regions spanned more than 40 268 CpGs.

269 Since the sliding windows are applied on the test statistic and not on the raw data, they are not as prone to many of the challenges the other methods face, such as probe bias for the methylation microarrays, or varying depth in sequencing studies, which all can be accounted

272 for in the first step of the modeling. Both DMRcate and bumphunter use very specific models 273 to evaluate point-wise methylation, leaving few options for the user to apply more complex 274 designs, like repeated measures, non-linear effects, or logistic regression. This is in contrast to 275 DMRScan, which relies only on the summary statistic, and can be applied on the test statistics 276 from any model as long as the underlying distribution of the test statistic is approximately 277 normal. Additionally, since the marginal summary statistic only has to be calculated once for 278 DMRScan, covariates and confounders can be included without any notable increase in 279 computational time.

280 When doing whole genome bisulfite sequencing or reduced representation bisulfite 281 sequencing, the methylation data set can be substantially larger than that of chip data. Since 282 DMRcate and DMRScan do not use permutation, they are not affected by this issue as much 283 as bumphunter, where the computational time can be substantial.

284 The three compared methods use different approaches for constructing p-values for the 285 candidate DMRs. One possible solution, by DMRcate, is to report the minimum p-value, or to 
aggregate the p-values using Stouffer's method. Stouffer's method is a way of combining p-

287 values by adding the Z-score normalized by the length of the candidate DMRs. For highly

288 dependent p-values, this may induce inflation in the test statistic, if the sum is not weighted

289 accordingly[28]. Bumphunter uses the minimum p-values in each DMR as its region-wise p-

290 values, which often deflates the p-values. Moreover, an "adjusted p-value" based on a

291 permutation test is given for each region, which is much more conservative. For the

292 bumphunter implementation, Jaffe et al. acknowledge that the region-wise adjusted p-values

293 may not always be representative, and that care should be taken when interpreting the

294 findings[10]. By applying a sliding window to call DMRs, we can utilize a well-defined

295 framework to construct p-values for each DMR which are adjusted for multiple testing.

296 Unlike bumphunter and DMRcate, the regions detected by the DMRScan method are always

297 genome-wide significant for the false discovery level set by the user.

298 DMRcate

299 An important gain of the applicability of summary statistics in our approach is the possibility

300 to analyze data from already published DNA methylome studies separately or in a meta-

301 analysis setting. In most methylomic or genomic meta-analysis, the individual raw data from

302 each separate study are not accessible, but a summary test statistic for each locus can often be

303 obtained across the different studies. This can open a new opportunity for meta-analysis

304 efforts in identification of DMRs.

\section{Conclusion}

306 DMRScan is a data-driven approach which properly addresses the multiple-testing challenge 307 when claiming genome-wide significance for differentially methylated regions. DMRScan 
308 performs better in terms of power compared to previously introduced methods, while keeping

309 the false discovery rate controlled.

\section{List of abbreviations}

\begin{tabular}{|l|l|}
\hline AR $(\mathrm{p})$ & Autoregressive process of order $\mathrm{p}$ \\
\hline ChIP & Chromatin Immunoprecipitation \\
\hline DMR & Differentially methylated region \\
\hline$E_{k}$ & Expected number of significant windows of size $\mathrm{k}$ \\
\hline FDR & False discovery rate \\
\hline MCMC & Markov Chain Monte Carlo \\
\hline OU-process & Ornstein-Uhlenbeck process \\
\hline$t_{k}$ & Window threshold for sliding windows of size $\mathrm{k}$ \\
\hline
\end{tabular}

\section{Declarations}

\section{Ethics}

314 The Coordinating Ethics Committees of the Hospital Districts of Helsinki and Uusimaa

315 approved the study.

\section{Consent for publication:}

317 Informed consent was obtained from all participants and as well as one of their legal

318 guardians.

\section{Availability of data and materials}

320 The $\mathrm{R}$ package is placed at Bioconductor under the name DMRScan, along with the example

321 data set used in this paper. The R-code for comparing the methods is available by the author

322 upon request.

\section{Competing interests}

324 The authors declare that they have no competing interests 
326 This work was supported by the University of Oslo [grant number 531217/1231]; Folkhälsan

327 Research Foundation; The Academy of Finland [grant number 250704]; The Life and Health

328 Medical Fund [grant number 1-23-28]; The Swedish Cultural Foundation in Finland [grant

329 number 15/0897]; The Signe and Ane Gyllenberg Foundation [grant number 37-1977-43];

330 and The Yrjö Jahnsson Foundation [grant number 11486].

\section{Author Contributions}

CMP; conceived the experiment, made the R scripts, did the analysis, wrote the paper LV; conceived the experiment, made the R scripts, did the analysis, wrote the paper TBR; supplied methylation values for the experiment, critically reviewed the manuscript $\mathrm{HFH}$; contributed to idea and funding, critically reviewed the manuscript BKA; conceived the experiment, did the analysis, wrote the paper All authors read and approved the final version of the manuscript.

\section{Acknowledgement}

We acknowledge Folkhälsan Research Center and the Fin-HIT study group: Sabina Simola, Stephanie Von Kreamer, Jesper Skand, Catharina Sarkkola, Sajan Raju and Elisabete Weiderpass (Helsinki, Finland) for providing data for benchmarking the different models. Institute for Molecular Medicine Finland (FIMM) provided computational infrastructure and preformed the sequencing to this project. Suzanne Campbell and Marissa LaBlanc for critical evaluation of this manuscript.

\section{References}

1. Laurent L, Wong E, Li G, Huynh T, Tsirigos A, Ong CT, Low HM, Kin Sung KW, Rigoutsos I, Loring $\mathrm{J}$ et al: Dynamic changes in the human methylome during differentiation. Genome research 2010, 20(3):320-331. 
2. Peters TJ, Buckley MJ, Statham AL, Pidsley R, Samaras K, R VL, Clark SJ, Molloy PL: De novo identification of differentially methylated regions in the human genome. Epigenetics \& chromatin 2015, 8:6.

3. Jones PA: Functions of DNA methylation: islands, start sites, gene bodies and beyond. Nature reviews Genetics 2012, 13(7):484-492.

4. Rakyan VK, Down TA, Balding DJ, Beck S: Epigenome-wide association studies for common human diseases. Nature reviews Genetics 2011, 12(8):529-541.

5. Sun YV, Levin AM, Boerwinkle E, Robertson H, Kardia SL: A scan statistic for identifying chromosomal patterns of SNP association. Genetic Epidemiology 2006, 30(7):627-635.

6. Feinberg AP, Irizarry RA, Fradin D, Aryee MJ, Murakami P, Aspelund T, Eiriksdottir G, Harris TB, Launer L, Gudnason V et al: Personalized epigenomic signatures that are stable over time and covary with body mass index. Sci Transl Med 2010, 2(49):49ra67.

7. Reiner-Benaim A, Davis RW, Juneau K: Scan statistics analysis for detection of introns in time-course tiling array data. Statistical applications in genetics and molecular biology 2014, 13(2):173-190.

8. Slieker RC, Bos SD, Goeman JJ, Bovee JV, Talens RP, van der Breggen R, Suchiman $\mathrm{HE}$, Lameijer EW, Putter H, van den Akker EB et al: Identification and systematic annotation of tissue-specific differentially methylated regions using the Illumina 450k array. Epigenetics \& chromatin 2013, 6(1):26.

9. Hansen KD, Langmead B, Irizarry RA: BSmooth: from whole genome bisulfite sequencing reads to differentially methylated regions. Genome biology 2012, 13(10):R83.

10. Jaffe AE, Murakami P, Lee H, Leek JT, Fallin MD, Feinberg AP, Irizarry RA: Bump hunting to identify differentially methylated regions in epigenetic epidemiology studies. Int J Epidemiol 2012, 41(1):200-209.

11. Butcher LM, Beck S: Probe Lasso: a novel method to rope in differentially methylated regions with 450K DNA methylation data. Methods 2015, 72:21-28.

12. Shen L, Zhu J, Robert Li S-Y, Fan X: Detect differentially methylated regions using non-homogeneous hidden Markov model for methylation array data.

Bioinformatics 2017, 33(23):3701-3708.

13. Korthauer K, Chakraborty S, Benjamini Y, Irizarry RA: Detection and accurate False Discovery Rate control of differentially methylated regions from Whole Genome Bisulfite Sequencing. bioRxiv 2017:183210.

14. Lun AT, Smyth GK: csaw: a Bioconductor package for differential binding analysis of ChIP-seq data using sliding windows. Nucleic acids research 2015, 44(5):e45-e45.

15. Rozowsky J, Euskirchen G, Auerbach RK, Zhang ZD, Gibson T, Bjornson R, Carriero $\mathrm{N}$, Snyder M, Gerstein MB: PeakSeq enables systematic scoring of ChIP-seq experiments relative to controls. Nature biotechnology 2009, 27(1):66-75.

16. Benjamini Y, Taylor J, Irizarry RA: Selection Corrected Statistical Inference for Region Detection with High-throughput Assays. bioRxiv 2016:082321.

17. Stouffer SA, Suchman EA, DeVinney LC, Star SA, Williams RM, Lumsdaine AA, Lumsdaine MH, Smith MB, Janis IL, Cottrell LS: Studies in Social Psychology in World War II.: The American Soldier: Adjustment During Army Life; 1965.

18. Zhang Y: Poisson approximation for significance in genome-wide ChIP-chip tiling arrays. Bioinformatics 2008, 24(24):2825-2831. 
19. Ritchie ME, Phipson B, Wu D, Hu Y, Law CW, Shi W, Smyth GK: limma powers differential expression analyses for RNA-sequencing and microarray studies. Nucleic acids research 2015:gkv007.

20. Satterthwaite FE: An approximate distribution of estimates of variance

components. Biometrics bulletin 1946, 2(6):110-114.

21. Aldous D: Probability approximations via the Poisson clumping heuristic, vol. 77 : Springer Science \& Business Media; 1989.

22. Siegmund DO, Zhang NR, Yakir B: False discovery rate for scanning statistics. Biometrika 2011, 98(4):979-985.

23. Siegmund D: Sequential analysis: tests and confidence intervals: Springer Science \& Business Media; 1985.

24. Siegmund D, Yakir B: The statistics of gene mapping: Springer Science \& Business Media; 2007.

25. Rounge TB, Page CM, Lepisto M, Ellonen P, Andreassen BK, Weiderpass E: Genome-wide DNA methylation in saliva and body size of adolescent girls. Epigenomics 2016, 8(11):1495-1505.

26. Du P, Zhang X, Huang CC, Jafari N, Kibbe WA, Hou L, Lin SM: Comparison of Beta-value and $M$-value methods for quantifying methylation levels by microarray analysis. BMC bioinformatics 2010, 11:587.

27. Bock C: Analysing and interpreting DNA methylation data. Nature Reviews Genetics 2012, 13(10):705-719.

28. Peng G, Luo L, Siu H, Zhu Y, Hu P, Hong S, Zhao J, Zhou X, Reveille JD, Jin L: Gene and pathway-based second-wave analysis of genome-wide association studies. European Journal of Human Genetics 2010, 18(1):111-117. 
422 Table 1 Comparison of the parameters between the five models used in the benchmarking.

423 Figure 1 Comparison of the convergence in power for all five methods, as well as the false 424 positive rate, both as a function of increasing effect size. Top panel (A-B) represents the 425 power to detect causal DMRs for the two different scenarios. The lower panel (C-D) 426 represents the power to detect CpGs within a causal DMR. The dashed lines represent false 427 positives. Bumphunter and DMRScan (Siegmund) had a very similar false positive rate 428 cannot be distinguished as they are directly on top of each other. 